\title{
An Atypical Presentation of a Patient with Renal Abscess
}

\author{
Blaine $C^{1}$, Annaliese Elam ${ }^{1}$, Yee $\mathrm{G}^{2}$ and Ranasinghe $\mathrm{L}^{3 *}$ \\ ${ }^{1}$ Fourth-year medical student California Northstate University College of Medicine (CNUCOM), USA \\ ${ }^{2}$ Emergency physician, CNUCOM, USA \\ ${ }^{3}$ Professor and Clerkship Director for Emergency Medicine, CNUCOM, USA
}

*Corresponding author: Leonard Ranasinghe, MD, Professor and Clerkship Director for Emergency Medicine, California Northstate University College of Medicine, 9700 W. Taron Dr., Elk Grove, California 95757, USA.

To Cite This Article: Blaine C, Annaliese Elam, Yee G, Ranasinghe L. An Atypical Presentation of a Patient with Renal Abscess. Am J Biomed Sci \& Res. 2021 - 13(6). AJBSR.MS.ID.001939. DOI: 10.34297/AJBSR.2021.13.001939.

Received: 跙 August 11, 2021; Published: 眥 August 24, 2021

\section{Introduction}

Renal and perirenal abscesses are two uncommon diagnoses that carry the potential for significant mortality, but those entities often present with vague and nonspecific symptoms as demonstrated with our patient. In earlier decades, case fatality rates for these conditions were as high as $39-50 \%$ [1]. More recent studies have shown mortality rates ranging from $0-7 \%$ [2]. Incidence is presently estimated at $1-10$ per 10,000 admissions, and increases with predisposing conditions such as diabetes mellitus, obstructing stones, and other urinary tract abnormalities [3]. The most common risk factor for both conditions has consistently been shown to be diabetes mellitus $[2,4]$.

The pathophysiology of renal and perirenal abscesses involves ascending infection of the urinary tract, largely by gram-negative bacilli, which is the likely cause for our patient. The alternative is hematogenous spread from distal sites of infection, the majority of which is caused by staphylococcal species. Renal abscesses are confined to the cortex or medulla of the kidneys, whereas perirenal abscesses involve infection that has spread to the space between the renal capsule and Gerota's fascia. 10\% of renal cortical abscesses will lead to perinephric abscesses [4], and many patients present with mixed renal and perirenal disease. As compared to perirenal abscesses, renal abscesses were more likely to be caused by enteric gram-negative bacteria, specifically E. coli and K. pneumonia [2] as was seen with our patient.

Multiple studies have demonstrated that the most common presenting complaints include fever, chills, and flank pain or tenderness [5], but only a minority will have the typical indication of a urinary tract infection with dysuria [2]. However, given that ascending infections are the typical source, a history of symptoms may be key to diagnosis. The average duration of fever in these patients is 10-14 days [2,5] which indicates a deeper infection. Lab work commonly reveals leukocytosis with left shift [2]. Predictors of poor prognosis include increased age, lethargy as a presenting complaint and impaired kidney function, as evidenced by elevated BUN [5].

\section{Case Presentation}

Our patient was an 86-year-old female with a history of insulin-independent diabetes mellitus who presented to the Emergency Department by ambulance from home with complaints of generalized weakness and diarrhea that started 10 days ago after beginning a new course of antibiotics for a recent urinary tract infection. She reported having 10 watery, brown stools per day without blood or mucus. She also reported a foul odor to her urine without dysuria or hematuria. She further reported a cramping spasm of the muscle in her right hand, chronic right lower abdominal pain, and chronic back pain. She denied fever, nausea, vomiting, shortness of breath, chest pain, focal weakness, numbness, and tingling. Per review of the patient's records, urine culture from her previous hospital visit grew out Klebsiella and she was prescribed cefadroxil 500mg BID for 10 days which she reported completing. She reported multiple recurrences of urinary tract infections in the last 6 months, including 2 which were treated at this Emergency Department. 
Examination revealed a comfortable appearing 86-year-old female who was fully alert and oriented and able to provide an extensive history. Vital signs showed temperature of 37.6C, heart rate of $80 \mathrm{bpm}$, respiratory rate of $20 \mathrm{rpm}$, blood pressure of 136/61, and oxygen saturation of $95 \%$ on room air. Patient had dry oral mucosa with poor skin turgor. Auscultation revealed an irregularly irregular heart rhythm and vesicular lung sounds bilaterally. Abdomen was soft and nontender with normoactive bowel sounds and mild left costovertebral angle tenderness. She had no focal motor or sensory deficits.

Our initial impression of this patient was a non-toxic but dehydrated elderly female who was suffering from diarrhea after multiple courses of antibiotics. Her multiple courses of antibiotics for urinary tract infections raised concerns for $C$. difficile diarrhea, antibiotic resistant urinary tract infection, pyelonephritis, renal abscess, or even a vesicoenteric fistula. However, she had no other significant medical history beyond hypertension and diabetes and no major surgical history. A more severe infection such as $C$. difficile, pyelonephritis, or renal abscess seemed unlikely given her absence of fever and otherwise normal vital. Her muscle spasms and complaints of weakness appeared to be related to electrolyte abnormalities from her diarrhea. Complete blood count, metabolic panel, troponin, electrocardiogram, lactic acid, C. difficile toxin and stool studies, blood culture, urinalysis, chest X-ray, and computed tomography of the abdomen were ordered. Given her concomitant abdominal and back pain, a CT was determined to be the most efficient first-line imaging.

Laboratory studies showed an elevated white blood cell count at 19.6 , sodium of 132 , potassium 3.3 , chloride 94 , bicarbonate 24 , BUN 20, creatinine 0.73 , and glucose 124 . Other electrolytes and liver function tests were within normal limits. Lactate and troponin were not elevated. Stool was negative for $C$. difficile toxins. Chest $\mathrm{X}$-ray was unremarkable. EKG showed new onset atrial fibrillation at a rate of $94 \mathrm{bpm}$. Urinalysis showed a large amount of leukocyte esterase with $4+$ bacteria. CT abdomen from 1 year prior showed "multiple exophytic cysts of bilateral kidneys with hyperdensity measuring $4 \mathrm{~mm}$ within the midpole of the right kidney." Imaging taken on the day of her visit is included below (Figure 1).

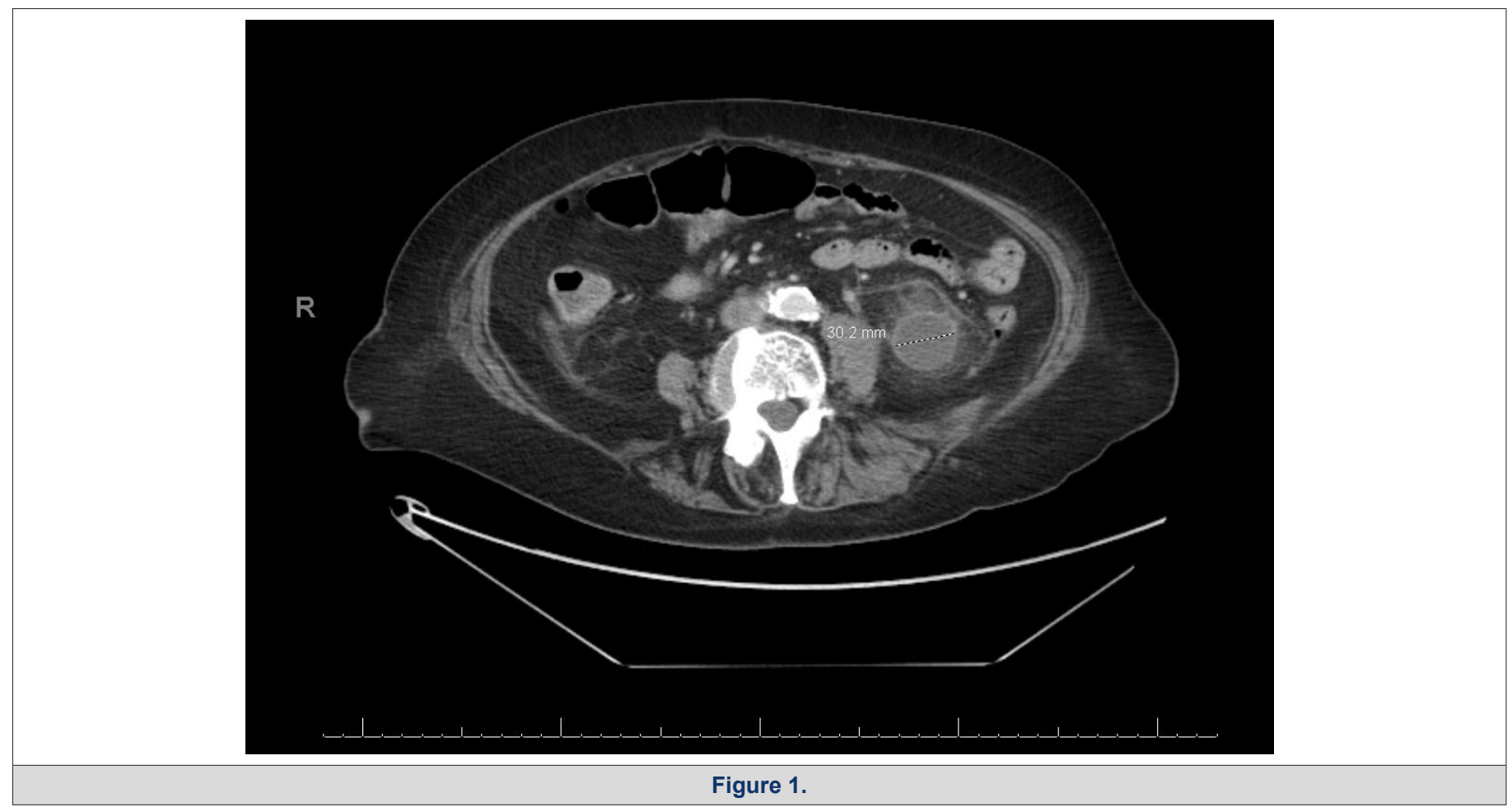

Radiology described the findings as "multiple bilateral renal cysts noted. Largest at the lower pole on the left measures up to $3 \mathrm{~cm}$, with peripheral enhancement and surrounding inflammation noted possibly infectious process/developing abscess."

\section{Management and Outcome}

Patient was initially started on IV fluids for dehydration upon arrival in the ED. Given her vital signs, suspicion for sepsis was low so antibiotics were held until a cause of her symptoms became apparent. Once imaging was available showing the source of infection, the patient was started on piperacillin with tazobactam. Urology was consulted and recommended that the patient be admitted for drainage of the abscess by interventional radiology with continuation of her prescribed antibiotic regimen.

Our patient was admitted to the hospital for 3 days. Interventional radiology was able to aspirate $17 \mathrm{~mL}$ of purulent 
fluid from the abscess. Urine culture, blood culture, and aspirate culture all grew out Klebsiella pneumonia.

She was discharged with a 3 -week course of ceftriaxone $2 \mathrm{~g}$ daily and plan for a repeat CT abdomen at 3 weeks. Follow-up
CT showed improvement of the abscess. Radiology described the findings as "Significant interval decrease in size of a bilobed left lower pole thick-walled cystic lesion measuring up to $1.6 \mathrm{~cm}$ transverse, previously $3 \mathrm{~cm}$. Adjacent perirenal fat stranding is also significantly decreased from previous exam" (Figure 2).

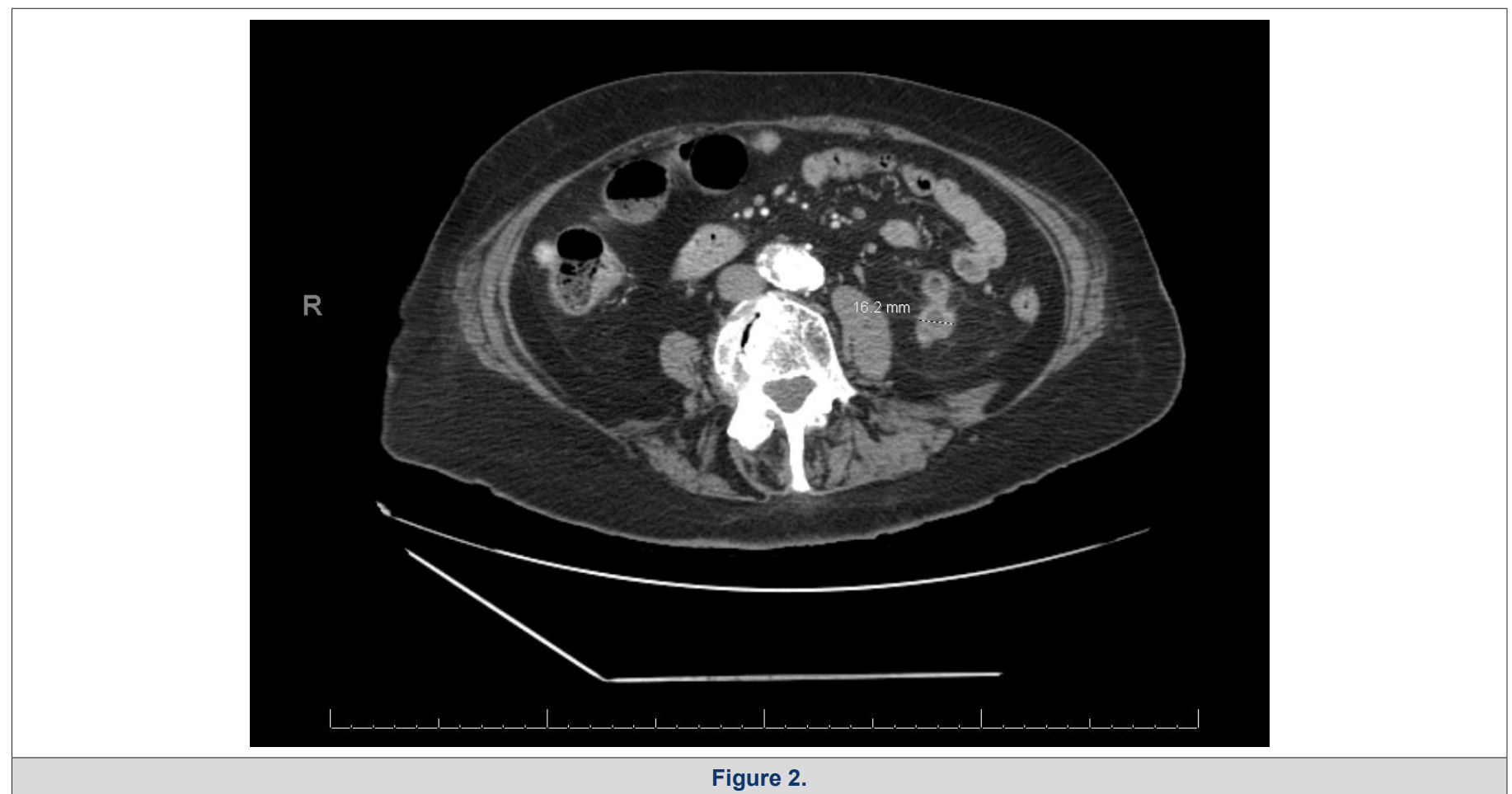

\section{Conclusion}

It is important to have a high index of clinical suspicion for renal and perirenal abscesses in the emergency department, as early diagnosis and antibiotic therapy have led to significant decreases in their associated morbidity and mortality. A review of the literature indicates that aggressive early imaging with ultrasound or CT has been a major contributor for improved clinical outcomes. In this case, the patient appeared well with largely normal vital signs, though her history of diabetes mellitus and recent recurrent urinary tract infections lowered our threshold to obtain abdominal imaging. Thus, we were able to diagnosis and treat her abscess before more significant complications arose.

\section{References}

1. Ko M C, Liu C C, Liu C K, Woung L C, Chen H F, et al. (2018) Incidence of renal and perinephric abscess in diabetic patients: a population-based national study. Epidemiol Infect 139(2): 229-235.

2. Lee B E, Seol H Y, Kim T K, Seong E Y, Song S H, et al. (2008) Recent clinical overview of renal and perirenal abscesses in 56 consecutive cases. Korean J Intern Med 23(3): 140-148.

3. Benson Aaron (2021) Renal Corticomedullary Abscess. Practice Essentials, Pathophysiology, Etiology.

4. Rai R S, Karan S C, Kayastha A (2007) Renal and Perinephric Abscesses Revisited. Med J Armed Forces India 63(3): 223-225.

5. Yen D H, Hu S C, Tsai J, Kao W F, Chern C H, et al. (1999) Renal abscess: Early diagnosis and treatment. Am J Emerg Med 17(2): 192-197. 